\title{
A New Pre-loaded Membrane Geometric Stiffness Matrix with Full Rigid Body Capabilities
}

\author{
Paul A. Bosela \\ Cleveland State University, p.bosela@csuohio.edu \\ D. R. Ludwiczak \\ NASA Lewis Research Center
}

Follow this and additional works at: https://engagedscholarship.csuohio.edu/encee_facpub

Part of the Structures and Materials Commons

How does access to this work benefit you? Let us know!

\section{Publisher's Statement}

NOTICE: this is the author's version of a work that was accepted for publication in Computers \& Structures. Changes resulting from the publishing process, such as peer review, editing, corrections, structural formatting, and other quality control mechanisms may not be reflected in this document. Changes may have been made to this work since it was submitted for publication. A definitive version was subsequently published in Computers \& Structures, 60, 1 , (01-01-1996); 10.1016/0045-7949(95)60341-7

\section{Original Citation}

Bosela P. A., and Ludwiczak D. R. (1996). A New Pre-loaded Membrane Geometric Stiffness Matrix with Full Rigid Body Capabilities. Computers \& Structures, 60, 1, 159-168.

This Article is brought to you for free and open access by the Civil and Environmental Engineering at EngagedScholarship@CSU. It has been accepted for inclusion in Civil and Environmental Engineering Faculty Publications by an authorized administrator of EngagedScholarship@CSU. For more information, please contact library.es@csuohio.edu. 


\title{
A NEW PRE-LOADED MEMBRANE GEOMETRIC STIFFNESS MATRIX WITH FULL RIGID BODY CAPABILITIES
}

\author{
P. A. Bosela $†$ and D. R. Ludwiczak‡ \\ $\dagger$ Department of Engineering Technology, Cleveland State University, Cleveland, Ohio 44115, U.S.A. \\ ¥Engineering Directorate, Structural Systems Division, Dynamics Branch, \\ NASA Lewis Research Center, Cleveland, Ohio 44135, U.S.A.
}

\section{NOTATION}

$\begin{array}{ll}a & \text { membrane element length } \\ b & \text { membrane element width } \\ {[D F C]} & \text { directed force correction matrix } \\ {[D F C \text { Total }]} & \text { matrix of all corrections ([DFC]+[MCOR]) } \\ E & \text { Young's modulus (Modulus of elasticity) } \\ h & \text { element thickness } \\ {[K e]} & \text { elastic stiffness matrix } \\ {[K g]} & \text { geometric stiffness matrix } \\ \mathbf{K}_{i j} & i, j \text { element of stiffness matrix } \\ {[M]} & \text { mass matrix } \\ {[M C O R]} & \text { fixed end moment correction } \\ N & \text { distributed tensile load }\left(N_{x}=N \text { for example, }\right. \\ & \left.\text { where } N_{y}=N_{x y}=0 .\right) \\ N_{x} & \text { distributed tensile load in } x \text {-direction } \\ N_{y} & \text { distributed tensile load in } y \text {-direction } \\ N_{x y} & \text { distributed shear load on membrane } \\ \alpha_{i} & \text { element constant } \\ \beta_{i} & \text { element constant } \\ \rho & \text { mass density } \\ v & \text { Poisson's ratio }\end{array}$

\section{INTRODUCTION}

Due to economic constraints associated with payload cost, space structures must consist of light-weight, and subsequently relatively flexible, components. The total stiffness of a member, in many cases, includes both the elastic stiffness, due to the material properties and member configuration, as well as geometric or initial stress stiffness due to pre-loads. Accurate prediction of the natural frequencies and mode shapes is essential for determining the adequacy of components, and for designing a controls system.
The finite element method is normally used to perform this analysis. However, a phenomenon known as "grounding" or false stiffening, occurs when the geometric stiffness matrix is used. When a pre-loaded model with free/free boundary conditions is analyzed, it behaves as if it is restrained internally. Thus, it is unable to rotate as a rigid body. Instead, erroneous "pseudo-forces" develop during rigid body motion. Free vibration analysis also yields an erroneous (non-zero) natural frequency associated with rigid body rotation. Bosela et al. [1-3] have examined the grounding phenomenon for a pre-loaded beam, and determined that it is caused by a force imbalance during rigid body rotation, and is typical of beam geometric stiffness matrices formulated by others, even those which include higher order effects. By utilizing a directed force premise, and extending to a global model the natural mode approach used by Argyris and Symeonidis [4] for developing load correction factors for non-conservative forces, a preloaded beam geometric stiffness matrix with full rigid body capabilities was developed and tested $[3,5]$.

This paper has the following objectives:

(1) Examine the grounding phenomenon as it relates to pre-loaded membrane elements.

(2) Develop a pre-loaded membrane element with full rigid body capabilities by following the directed force approach used successfully in developing the pre-loaded beam element. 


\section{MEMBRANE ELEMENTS}

Although various membrane elements are used in commercial finite element programs, most are not readily available in explicit form. One which is stated explicitly by Yang [6] is the four-node rectangular element in Fig. 1, which has either 12 or 16 degrees of freedom (DOF). The degrees of freedom numbered as $13,14,15$ and 16 , correspond to second-order twist terms such as $\partial^{2} w / \partial x \partial y$. This element is obtained by combining the shape functions for the elementary beam element in both the $x$ and $y$ directions.

The geometric, or initial stress stiffness matrix $[\mathrm{Kg}]$ for this plate element when the distributed load is in the $x$-direction only is

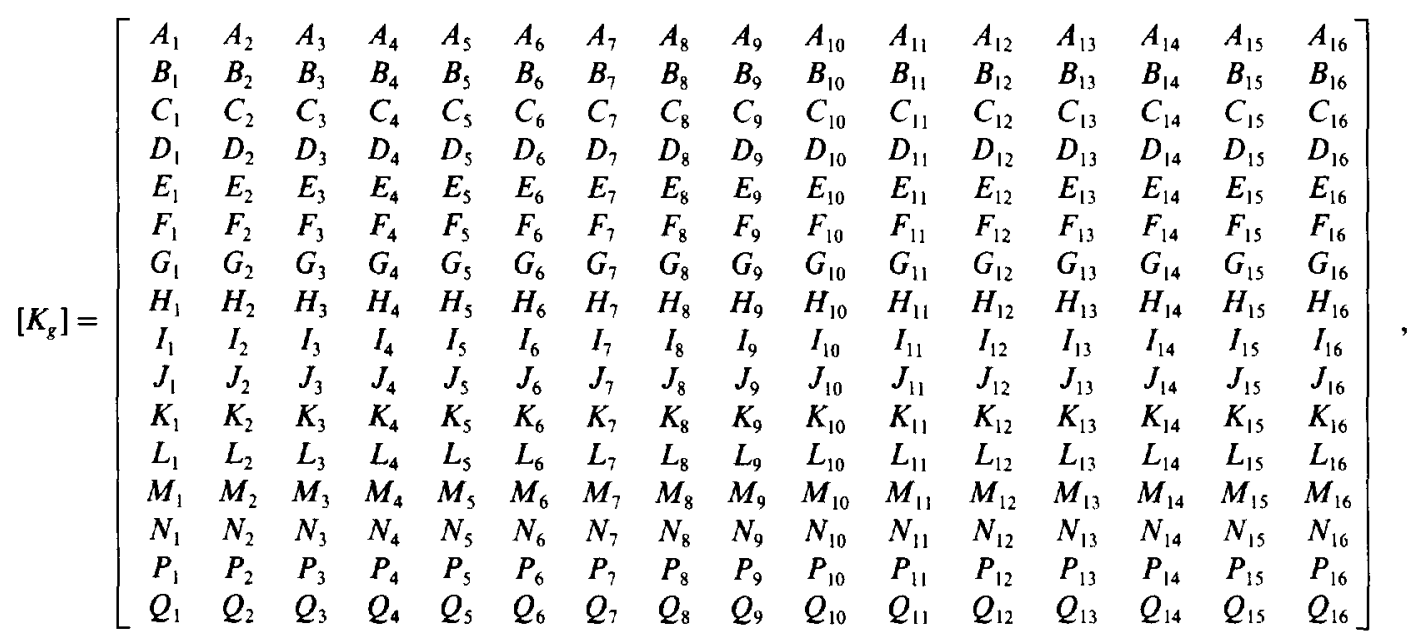

$$
\begin{aligned}
& A_{1}=B_{2}=C_{3}=D_{4}=\frac{78 N_{x} b}{175 a} \quad A_{2}=B_{1}=C_{4}=D_{3}=-\frac{78 N_{x} b}{175 a} \\
& A_{4}=B_{3}=C_{2}=D_{1}=\frac{27 N_{x} b}{175 a} \quad A_{3}=B_{4}=C_{1}=D_{2}=-\frac{27 N_{x} b}{175 a} \\
& A_{5}=A_{6}=D_{7}=D_{8}=\frac{13 N_{x} b}{350} \quad B_{5}=B_{6}=C_{7}=C_{8}=-\frac{13 N_{x} b}{350} \\
& A_{7}=A_{8}=D_{5}=D_{6}=\frac{9 N_{x} b}{700} \quad B_{7}=B_{8}=C_{5}=C_{6}=-\frac{9 N_{x} b}{700} \\
& A_{9}=B_{10}=C_{12}=D_{11}=\frac{11 N_{x} b^{2}}{175 a} \quad A_{10}=B_{9}=C_{11}=D_{12}=-\frac{11 N_{x} b^{2}}{175 a} \\
& A_{11}=B_{12}=C_{10}=D_{9}=\frac{13 N_{x} b^{2}}{350 a} \quad A_{12}=B_{11}=C_{9}=D_{10}=-\frac{13 N_{x} b^{2}}{350 a} \\
& A_{13}=A_{14}=C_{15}=C_{16}=\frac{11 N_{x} b^{2}}{2100} \quad B_{13}=B_{14}=D_{15}=D_{16}=-\frac{11 N_{x} b^{2}}{2100} \\
& B_{15}=B_{16}=D_{13}=D_{14}=\frac{13 N_{x} b^{2}}{4200} \quad A_{15}=A_{16}=C_{13}=C_{14}=-\frac{13 N_{x} b^{2}}{4200} \\
& E_{1}=F_{1}=G_{4}=H_{4}=\frac{13 N_{x} b}{350} \quad E_{2}=F_{2}=G_{3}=H_{3}=-\frac{13 N_{x} b}{350}
\end{aligned}
$$



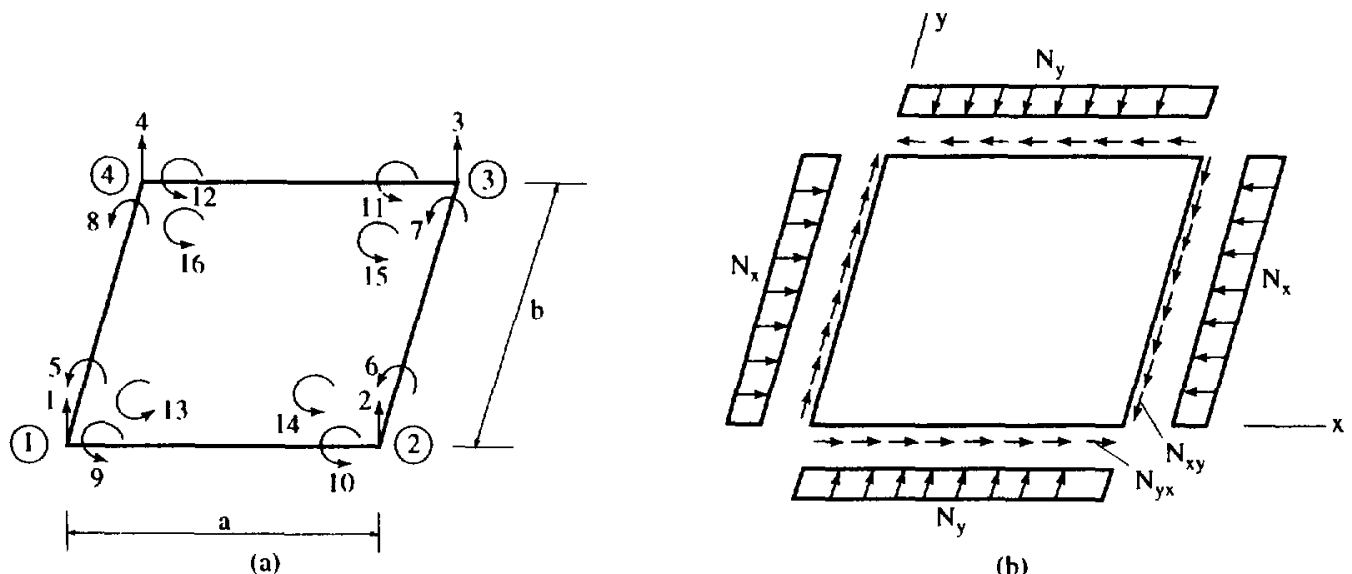

(b)

Fig. 1.(a) Four-node rectangular plate element with 16 DOF. (b) In-plane normal and shear forces on quadrilateral membrane element.

$$
\begin{aligned}
& E_{4}=F_{4}=G_{1}=H_{1}=\frac{9 N_{x} b}{700} \quad E_{3}=F_{3}=G_{2}=H_{2}=-\frac{9 N_{x} b}{700} \\
& E_{5}=F_{6}=G_{7}=H_{8}=\frac{26 N_{x} a b}{525} \quad E_{6}=F_{5}=G_{8}=H_{7}=-\frac{13 N_{x} a b}{1050} \\
& E_{7}=F_{8}=G_{5}=H_{6}=-\frac{3 N_{x} a b}{700} \quad E_{8}=F_{7}=G_{6}=H_{5}=\frac{3 N_{x} a b}{175} \\
& E_{9}=F_{9}=G_{11}=H_{11}=\frac{11 N_{x} b^{2}}{2100} \quad E_{10}=F_{10}=G_{12}=H_{12}=-\frac{11 N_{x} b^{2}}{2100} \\
& E_{11}=F_{11}=G_{9}=H_{9}=\frac{13 N_{x} b^{2}}{4200} \quad E_{12}=F_{12}=G_{10}=H_{10}=-\frac{13 N_{x} b^{2}}{4200} \\
& E_{13}=F_{14}=\frac{11 N_{x} a b^{2}}{1575} \quad G_{15}=H_{16}=-\frac{11 N_{x} a b^{2}}{1575} \\
& G_{16}=H_{15}=\frac{11 N_{x} a b^{2}}{6300} \quad E_{14}=F_{13}=-\frac{11 N_{x} a b^{2}}{6300} \\
& I_{5}=I_{6}=J_{7}=K_{8}=\frac{11 N_{x} b^{2}}{2100} \quad J_{5}=J_{6}=L_{1}=L_{8}=-\frac{11 N_{x} b^{2}}{2100} \\
& E_{15}=F_{16}=\frac{13 N_{x} a b^{2}}{12600} \quad G_{13}=H_{14}=-\frac{13 N_{x} a b^{2}}{12600} \\
& G_{14}=H_{13}=\frac{13 N_{x} a b^{2}}{3150} \quad E_{16}=F_{15}=-\frac{13 N_{x} a b^{2}}{3150} \\
& I_{2}=J_{1}=K_{3}=L_{4}=-\frac{11 N_{x} b^{2}}{175 a}
\end{aligned}
$$




$$
\begin{aligned}
& I_{7}=I_{8}=K_{5}=K_{6}=\frac{13 N_{x} b^{2}}{4200} \quad J_{7}=J_{8}=L_{5}=L_{6}=-\frac{13 N_{x} b^{2}}{4200} \\
& I_{9}=J_{10}=K_{11}=L_{12}=\frac{2 N_{x} b^{3}}{175 a} \quad I_{10}=K_{12}=L_{11}=J_{9}=-\frac{2 N_{x} b^{3}}{175 a} \\
& I_{11}=J_{12}=K_{9}=L_{10}=\frac{3 N_{x} b^{3}}{350 a} \quad I_{12}=J_{11}=K_{10}=L_{9}=-\frac{3 N_{x} b^{3}}{350 a} \\
& I_{13}=I_{14}=L_{15}=L_{16}=M_{9}=N_{9}=Q_{12}=P_{12}=\frac{N_{x} b^{3}}{1050} \\
& J_{13}=J_{14}=K_{15}=K_{16}=M_{10}=P_{11}=Q_{11}=-\frac{N_{x} b^{3}}{1050} \\
& J_{15}=J_{16}=K_{13}=K_{14}=M_{11}=N_{11}=P_{10}=Q_{10}=\frac{N_{x} b^{3}}{1400} \\
& I_{15}=I_{16}=L_{13}=L_{14}=M_{12}=N_{12}=P_{9}=Q_{9}=-\frac{N_{x} b^{3}}{1400} \\
& M_{1}=N_{1}=P_{3}=Q_{3}=\frac{11 N_{x} b^{2}}{2100} \quad M_{2}=N_{2}=P_{4}=Q_{4}=-\frac{11 N_{x} b^{2}}{2100} \\
& M_{4}=N_{4}=P_{2}=Q_{2}=\frac{13 N_{x} b^{2}}{4200} \quad M_{3}=N_{3}=P_{1}=Q_{1}=-\frac{13 N_{x} b^{2}}{4200} \\
& M_{5}=N_{6}=\frac{11 N_{x} a b^{2}}{1575} \quad M_{6}=N_{5}=-\frac{11 N_{x} a b^{2}}{6300} \\
& M_{8}=N_{7}=\frac{13 N_{x} a b^{2}}{3150} \quad M_{7}=N_{8}=-\frac{13 N_{x} a b^{2}}{12600} \\
& M_{13}=N_{14}=P_{15}=Q_{16}=\frac{2 N_{x} a b^{3}}{1575} \quad M_{14}=N_{13}=P_{16}=Q_{15}=-\frac{N_{x} a b^{3}}{3150} \\
& M_{15}=N_{16}=P_{13}=Q_{14}=\frac{N_{x} a b^{3}}{4200} \quad M_{16}=N_{15}=P_{14}=Q_{13}=-\frac{N_{x} a b^{3}}{1050} \\
& P_{5}=Q_{6}=\frac{13 N_{x} a b^{2}}{12600} \quad N_{10}=-\frac{N_{x} b^{3}}{1050} \quad P_{6}=Q_{5}=-\frac{13 N_{x} a b^{2}}{3150} \\
& P_{8}=Q_{7}=\frac{11 N_{x} a b^{2}}{6300} \quad P_{7}=Q_{8}=-\frac{11 N_{x} a b^{2}}{1575} .
\end{aligned}
$$

In order to solve for the natural frequencies, the corresponding four-node quadrilateral mass matrix developed by Yang was also used. The coefficients of the mass matrix are determined by using the equation

$$
\mathbf{M}_{i j}=\rho h \int_{0}^{b} \int_{0}^{a} f_{i}(x, y) f_{j}(x, y) \partial x \partial y .
$$




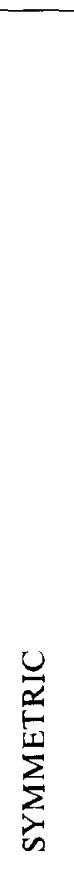

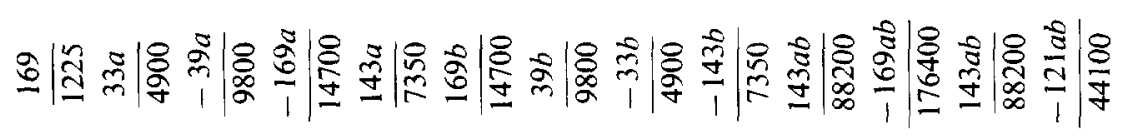

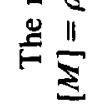




\section{GROUNDING OF MEMBRANE ELEMENTS}

Suppose one considers a free/free model with two 12 DOF elements, and a uniform tensile load applied in the $x$-direction only, as shown in Fig. 2. That element should possess five rigid body modes: (1) translation in the $y$-direction; (2) rigid body rotation about the $x$-axis; (3) rotation of the edge line between nodes 1,2 and 5 ; (4) rotation of the edge lines between nodes 4,3 and 6 ; (5) rigid body rotation about the $y$-axis. It should be noted that (2) and (4) only occur because for the example problem, there is no tension in the $y$-direction, and we are neglecting elastic stiffness effects.

In matrix form, assembling $[\mathrm{Kg}]$ and multiplying a matrix of these rigid body modes yields to rigid body rotation about the $y$-axis, was not present. Instead, pseudo-forces developed in degree of freedoms $1,4,9,12,13,14,17$ and 18 . The pseudo-forces in degrees of freedom 1, 4, 13 and 14 correspond to the forces required to keep the rotated model in equilibrium, which is consistent with the behavior of a pre-loaded beam element [2]. Hence, the procedure used successfully for the pre-loaded beam element [3] will be adapted.

The pseudo-moments developed in degrees of freedom $9,12,17$ and 18 did not occur with rotation of the pre-loaded beam element. It should be noted, however, that the form resembles a fixed-end moment caused by a distributed load.
$[K g] \cdot\left[\begin{array}{ccccc}1 & -1 & 0 & 0 & -1 \\ 1 & -1 & 0 & 0 & 0 \\ 1 & 1 & 0 & 0 & 0 \\ 1 & 1 & 0 & 0 & -1 \\ 0 & 0 & 0 & 0 & \frac{1}{a} \\ 0 & 0 & 0 & 0 & \frac{1}{a} \\ 0 & 0 & 0 & 0 & \frac{1}{a} \\ 0 & 0 & 0 & 0 & \frac{1}{a} \\ 0 & \frac{2}{b} & 1 & 0 & 0 \\ 0 & \frac{2}{b} & 1 & 0 & 0 \\ 0 & \frac{2}{b} & 0 & 1 & 0 \\ 1 & -1 & 0 & 0 & 1 \\ 1 & 1 & 0 & 0 & 1 \\ 0 & 0 & 0 & 0 & \frac{1}{a} \\ 0 & 0 & 0 & 0 & \frac{1}{a} \\ 0 & \frac{2}{b} & 1 & 0 & 0 \\ 0 & \frac{2}{b} & 0 & 1 & 0\end{array}\right]$

Examining the right hand side of eqn (5) reveals that rigid body modes 1-4 are present in the global $[K g]$. However, mode \#5, which corresponds

$=\left[\begin{array}{ccccc}0 & 0 & 0 & 0 & -\frac{N b}{2 a} \\ 0 & 0 & 0 & 0 & 0 \\ 0 & 0 & 0 & 0 & 0 \\ 0 & 0 & 0 & 0 & -\frac{N b}{2 a} \\ 0 & 0 & 0 & 0 & 0 \\ 0 & 0 & 0 & 0 & 0 \\ 0 & 0 & 0 & 0 & 0 \\ 0 & 0 & 0 & 0 & 0 \\ 0 & 0 & 0 & 0 & -\frac{N b^{2}}{12 a} \\ 0 & 0 & 0 & 0 & 0 \\ 0 & 0 & 0 & 0 & 0 \\ 0 & 0 & 0 & 0 & \frac{N b^{2}}{12 a} \\ 0 & 0 & 0 & 0 & \frac{N b}{2 a} \\ 0 & 0 & 0 & 0 & \frac{N b}{2 a} \\ 0 & 0 & 0 & 0 & 0 \\ 0 & 0 & 0 & 0 & 0 \\ 0 & 0 & 0 & 0 & \frac{N b^{2}}{12 a} \\ 0 & 0 & 0 & 0 & -\frac{N b^{2}}{12 a}\end{array}\right] .(5)$

DIRECTED-FORCE APPROACH

Consider Argyris's methodology [4] for the directed force problem. Let $\phi \cong\left(u_{4}-u_{18}\right) / 2 a$ or 


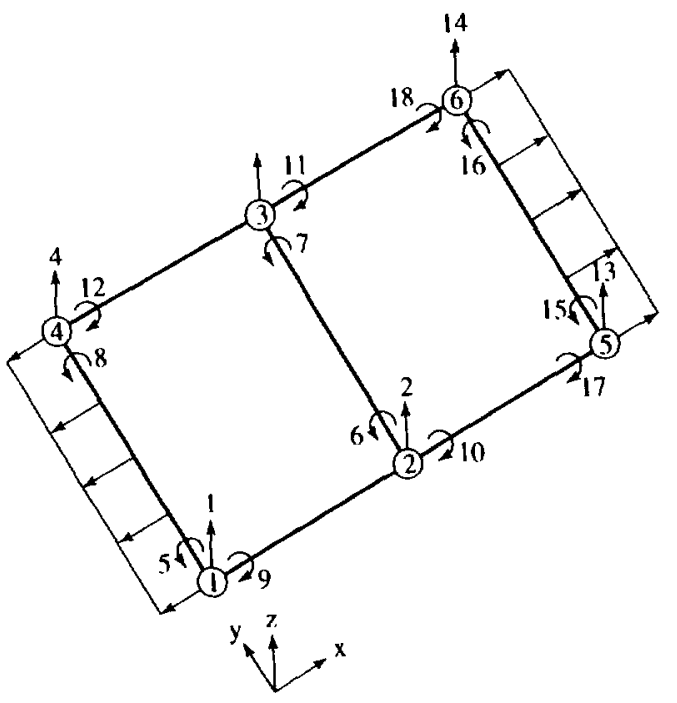

Fig. 2. Two element 18 DOF.

$\left(u_{1}-u_{17}\right) / 2 a$. If one neglects the change in the axial component during a small rotation, one obtains $N(b / 2) \cos \phi \cong N b / 2$. The load vector for this force

$\mathbf{R}^{\mathrm{DFC}}=\left[(N b / 2) \sin \left(u_{17}-u_{1} / 2 a\right) 00(N b / 2) \sin \right.$

$\times\left(u_{18}-u_{4} / 2 a\right) 00000000000000000$

$-(N b / 2) \sin \left(u_{17}-u_{1} / 2 a\right)$

$\left.-(N b / 2) \sin \left(u_{18}-u_{4} / 2 a\right) 00000000\right] . \quad(6)$

Taking

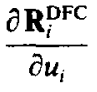

and assuming small angles yields

$$
\left[K^{\mathrm{DFC}}\right]=\left[\begin{array}{cccccccccccccccccc}
-b N & 0 & 0 & 0 & 0 & 0 & 0 & 0 & 0 & 0 & 0 & 0 & \frac{b N}{4 a} & 0 & 0 & 0 & 0 & 0 \\
0 & 0 & 0 & 0 & 0 & 0 & 0 & 0 & 0 & 0 & 0 & 0 & 0 & 0 & 0 & 0 & 0 & 0 \\
0 & 0 & 0 & 0 & 0 & 0 & 0 & 0 & 0 & 0 & 0 & 0 & 0 & 0 & 0 & 0 & 0 & 0 \\
0 & 0 & 0 & -\frac{b N}{4 a} & 0 & 0 & 0 & 0 & 0 & 0 & 0 & 0 & 0 & \frac{b N}{4 a} & 0 & 0 & 0 & 0 \\
0 & 0 & 0 & 0 & 0 & 0 & 0 & 0 & 0 & 0 & 0 & 0 & 0 & 0 & 0 & 0 & 0 & 0 \\
0 & 0 & 0 & 0 & 0 & 0 & 0 & 0 & 0 & 0 & 0 & 0 & 0 & 0 & 0 & 0 & 0 & 0 \\
0 & 0 & 0 & 0 & 0 & 0 & 0 & 0 & 0 & 0 & 0 & 0 & 0 & 0 & 0 & 0 & 0 & 0 \\
0 & 0 & 0 & 0 & 0 & 0 & 0 & 0 & 0 & 0 & 0 & 0 & 0 & 0 & 0 & 0 & 0 & 0 \\
0 & 0 & 0 & 0 & 0 & 0 & 0 & 0 & 0 & 0 & 0 & 0 & 0 & 0 & 0 & 0 & 0 & 0 \\
0 & 0 & 0 & 0 & 0 & 0 & 0 & 0 & 0 & 0 & 0 & 0 & 0 & 0 & 0 & 0 & 0 & 0 \\
0 & 0 & 0 & 0 & 0 & 0 & 0 & 0 & 0 & 0 & 0 & 0 & 0 & 0 & 0 & 0 & 0 & 0 \\
0 & 0 & 0 & 0 & 0 & 0 & 0 & 0 & 0 & 0 & 0 & 0 & 0 & 0 & 0 & 0 & 0 & 0 \\
b N & 0 & 0 & 0 & 0 & 0 & 0 & 0 & 0 & 0 & 0 & 0 & -\frac{b N}{4 a} & 0 & 0 & 0 & 0 & 0 \\
4 a & 0 & 0 & & & 0 & & & 0 & & 0 \\
0 & 0 & 0 & \frac{b N}{4 a} & 0 & 0 & 0 & 0 & 0 & 0 & 0 & 0 & 0 & -\frac{b N}{4 a} & 0 & 0 & 0 & 0 \\
0 & 0 & 0 & 0 & 0 & 0 & 0 & 0 & 0 & 0 & 0 & 0 & 0 & 0 & 0 & 0 & 0 & 0 \\
0 & 0 & 0 & 0 & 0 & 0 & 0 & 0 & 0 & 0 & 0 & 0 & 0 & 0 & 0 & 0 & 0 & 0 \\
0 & 0 & 0 & 0 & 0 & 0 & 0 & 0 & 0 & 0 & 0 & 0 & 0 & 0 & 0 & 0 & 0 & 0 \\
0 & 0 & 0 & 0 & 0 & 0 & 0 & 0 & 0 & 0 & 0 & 0 & 0 & 0 & 0 & 0 & 0 & 0
\end{array}\right] .
$$


Combining $[K g]+\left[K^{\mathrm{DFC}}\right]$ and multiplying by the rigid body mode matrix yields

$$
\left[[\mathrm{Kg}]+\left[\mathrm{K}^{\mathrm{DFC}}\right] \cdot\left[\begin{array}{ccccc}
0 & 0 & 0 & 0 & 0 \\
0 & 0 & 0 & 0 & 0 \\
0 & 0 & 0 & 0 & 0 \\
0 & 0 & 0 & 0 & 0 \\
0 & 0 & 0 & 0 & 0 \\
0 & 0 & 0 & 0 & 0 \\
0 & 0 & 0 & 0 & 0 \\
0 & 0 & 0 & 0 & 0 \\
0 & 0 & 0 & 0 & -\frac{N b^{2}}{12 a} \\
0 & 0 & 0 & 0 & 0 \\
0 & 0 & 0 & 0 & 0 \\
0 & 0 & 0 & 0 & \frac{N b^{2}}{12 a} \\
0 & 0 & 0 & 0 & 0 \\
0 & 0 & 0 & 0 & 0 \\
0 & 0 & 0 & 0 & 0 \\
0 & 0 & 0 & 0 & 0 \\
0 & 0 & 0 & 0 & \frac{N b^{2}}{12 a} \\
& 0 & 0 & 0 & -\frac{N b^{2}}{12 a}
\end{array}\right]\right.
$$

Note that the pseudo-force terms associated with degrees of freedom 1, 4, 13 and 14 have disappeared, but the pseudo-moment terms associated with degrees of freedom $9,12,17$ and 18 remain.

Once again, consider a rotation $\phi$ about the $y$-axis, and let $\phi=\left(u_{13}+u_{14}-u_{1}-u_{4}\right) / 4 a$. We have considered the equivalent concentrated vertical loads at the nodes due to the rotation in the determination of $\left[K^{\mathrm{DFC}}\right]$. However, this vertical component is really based on a distributed load. In order to replace it with concentrated loads at the node points, we must also include fixed-end moments. The load vector for the fixed end moments caused by the vertical distributed loads would be

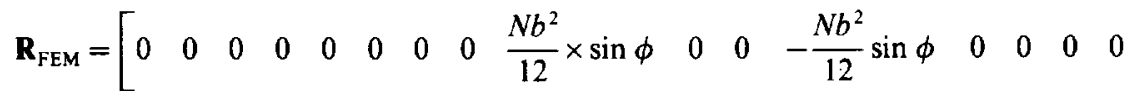

$$
\begin{aligned}
& \left.-\frac{N b^{2}}{12} \sin \phi \frac{n B^{2}}{12} \sin \phi\right]
\end{aligned}
$$

Taking $\frac{\partial R_{i}^{\mathrm{FEM}}}{\partial v_{i}}$, assuming small angles and enforcing symmetry yields 


\begin{tabular}{|c|c|c|c|c|c|c|c|c|c|c|c|c|c|c|c|c|c|}
\hline 0 & 0 & 0 & 0 & 0 & 0 & 0 & 0 & $-\frac{N b^{2}}{48 a}$ & 0 & 0 & $\frac{N b^{2}}{48 a}$ & 0 & 0 & 0 & 0 & $\frac{N b^{2}}{48 a}$ & $-\frac{N b^{2}}{48 a}$ \\
\hline 0 & 0 & 0 & 0 & 0 & 0 & 0 & 0 & 0 & 0 & 0 & 0 & 0 & 0 & 0 & 0 & 0 & 0 \\
\hline 0 & 0 & 0 & 0 & 0 & 0 & 0 & 0 & 0 & 0 & 0 & 0 & 0 & 0 & 0 & 0 & 0 & 0 \\
\hline 0 & 0 & 0 & 0 & 0 & 0 & 0 & 0 & $-\frac{N b^{2}}{48 a}$ & 0 & 0 & $\frac{N b^{2}}{48 a}$ & 0 & 0 & 0 & 0 & $\frac{N b^{2}}{48 a}$ & $-\frac{N b^{2}}{48 a}$ \\
\hline 0 & 0 & 0 & 0 & 0 & 0 & 0 & 0 & 0 & 0 & 0 & 0 & 0 & 0 & 0 & 0 & 0 & 0 \\
\hline 0 & 0 & 0 & 0 & 0 & 0 & 0 & 0 & 0 & 0 & 0 & 0 & 0 & 0 & 0 & 0 & 0 & 0 \\
\hline 0 & 0 & 0 & 0 & 0 & 0 & 0 & 0 & 0 & 0 & 0 & 0 & 0 & 0 & 0 & 0 & 0 & 0 \\
\hline 0 & 0 & 0 & 0 & 0 & 0 & 0 & 0 & 0 & 0 & 0 & 0 & 0 & 0 & 0 & 0 & 0 & 0 \\
\hline$-\frac{N b^{2}}{48 a}$ & 0 & 0 & $-\frac{N b^{2}}{48 a}$ & 0 & 0 & 0 & 0 & 0 & 0 & 0 & 0 & $\frac{N b^{2}}{48 a}$ & $\frac{N b^{2}}{48 a}$ & 0 & 0 & 0 & 0 \\
\hline 0 & 0 & 0 & 0 & 0 & 0 & 0 & 0 & 0 & 0 & 0 & 0 & 0 & 0 & 0 & 0 & 0 & 0 \\
\hline 0 & 0 & 0 & 0 & 0 & 0 & 0 & 0 & 0 & 0 & 0 & 0 & 0 & 0 & 0 & 0 & 0 & 0 \\
\hline$\frac{N b^{2}}{48 a}$ & 0 & 0 & $\frac{N b^{2}}{48 a}$ & 0 & 0 & 0 & 0 & 0 & 0 & 0 & 0 & $-\frac{N b^{2}}{48 a}$ & $-\frac{N b^{2}}{48 a}$ & 0 & 0 & 0 & 0 \\
\hline 0 & 0 & 0 & 0 & 0 & 0 & 0 & 0 & $\frac{N b^{2}}{48 a}$ & 0 & 0 & $-\frac{N b^{2}}{48 a}$ & 0 & 0 & 0 & 0 & $-\frac{N b^{2}}{48 a}$ & $\frac{N b^{2}}{48 a}$ \\
\hline 0 & 0 & 0 & 0 & 0 & 0 & 0 & 0 & $\frac{N b^{2}}{48 a}$ & 0 & 0 & $-\frac{N b^{2}}{48 a}$ & 0 & 0 & 0 & 0 & $-\frac{N b^{2}}{48 a}$ & $\frac{N b^{2}}{48 a}$ \\
\hline 0 & 0 & 0 & 0 & 0 & 0 & 0 & 0 & 0 & 0 & 0 & 0 & 0 & 0 & 0 & 0 & 0 & 0 \\
\hline 0 & 0 & 0 & 0 & 0 & 0 & 0 & 0 & 0 & 0 & 0 & 0 & 0 & 0 & 0 & 0 & 0 & 0 \\
\hline$\frac{N b^{2}}{48 a}$ & 0 & 0 & $\frac{N b^{2}}{48 a}$ & 0 & 0 & 0 & 0 & 0 & 0 & 0 & 0 & $-\frac{N b^{2}}{48 a}$ & $-\frac{N b^{2}}{48 a}$ & 0 & 0 & 0 & 0 \\
\hline$-\frac{N b^{2}}{48 a}$ & 0 & 0 & $-\frac{N b^{2}}{48 a}$ & 0 & 0 & 0 & 0 & 0 & 0 & 0 & 0 & $\frac{N b^{2}}{48 a}$ & $\frac{N b^{2}}{48 a}$ & 0 & 0 & 0 & 0 \\
\hline
\end{tabular}

At this point,

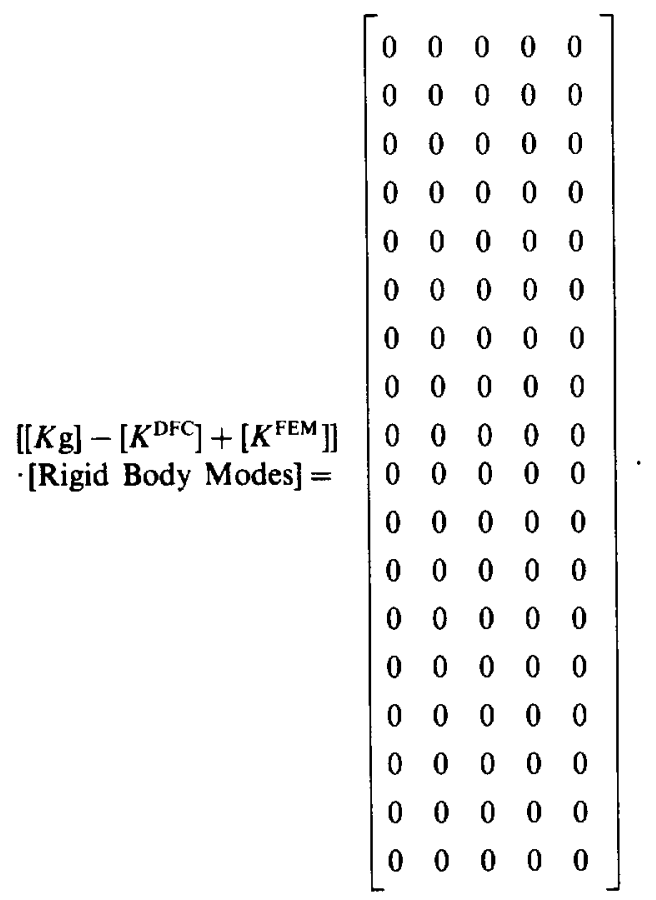

Thus, the all pseudo-forces and pseudo-moments have been eliminated.

\section{COMPARISON OF FREQUENCIES}

In order to determine the effect of the correction factors, a comparison of the first 12 natural frequencies of vibration was performed for the two element model. The results are included in Table 1.

Table 1. Comparison of frequencies of vibration for twoelement free/free model

\begin{tabular}{|c|c|c|c|c|}
\hline Frequency & $\begin{array}{c}\text { Yang's } \\
{[K g]_{16}} \\
\text { DOF }\end{array}$ & $\begin{array}{c}\text { Yang's } \\
{[K g] 24} \\
\text { DOF }\end{array}$ & $\begin{array}{l}\text { Corrected } \\
16 \mathrm{DOF}\end{array}$ & $\begin{array}{c}\text { Corrected } \\
24 \text { DOF }\end{array}$ \\
\hline 1 & 0 & 0 & 0 & 0 \\
\hline 2 & 0 & 0 & 0 & 0 \\
\hline 3 & 0 & 0 & 0 & 0 \\
\hline 4 & 0 & 0 & 0 & 0 \\
\hline 5 & 103.2 & 0 & 0 & 0.0152 \\
\hline 6 & 103.2 & 0 & 103.2 & 103.2 \\
\hline 7 & 118.0 & 0 & 118.0 & 103.2 \\
\hline 8 & 118.0 & 0 & 206.4 & 174.7 \\
\hline 9 & 206.4 & 0 & 206.4 & 206.4 \\
\hline 10 & 206.4 & 0 & 206.4 & 206.4 \\
\hline 11 & 206.4 & 0 & 206.4 & 206.4 \\
\hline 12 & 206.4 & 0 & 268.3 & 206.4 \\
\hline
\end{tabular}


As the table indicates, the missing rigid body mode (mode \#5, frequency $=0$ ), was obtained when the total directed force correction factors were added to Yang's $[\mathrm{Kg}]$.

\section{SUMMARY}

Based upon this investigation, the following conclusions can be made:

(1) Pre-loaded membrane elements exhibit the same "grounding" problems associated with preloaded beam elements. In other words, an internal grounding, or false stiffening, occurs when they are subjected to a rigid body rotation, which generates erroneous pseudo-forces.

(2) In addition to the pseudo-forces, erroneous pseudo-moments also develop during rigid body rotation.

(3) An erroneous non-zero frequency develops during rigid body rotation, instead of the required zero frequency associated with a rigid body mode.

(4) Using a directed force premise, and extending the Argyris approach for developing correction factors for non-conservative forces to the global level, the pseudo-forces were eliminated, but the pseudomoments still remained.

(5) By considering the fixed-end moments associated with the distributed load, and similarly developing correction factors, the pseudo-moments were eliminated, and the missing zero frequency associated with rigid body rotation were obtained.

(6) Future work should include comparison of results for larger models and with test results.

\section{REFERENCES}

1. P. Bosela, F. Shaker and D. Fertis, Dynamic analysis of space-related linear and non-linear structures. Comput. Struct. 44, 1145-1148 (1992).

2. P. Bosela, D. Fertis and F. Shaker, Grounding of space structures. Comput. Struct. 45, 143-153 (1992).

3. P. Bosela, D. Fertis and F. Shaker, A new pre-loaded beam geometric stiffness matrix with full rigid body capabilities. Comput. Struct. 45, 155-163 (1992).

4. J. Argyris and Sp. Symeonidis, Nonlinear finite element analysis of elastic systems under non-conservative loading-natural formulation. Part 1. Quasistatic problems. Comput. Meth. appl. Mech. Engng 26, 75-123 (1981).

5. D. Fertis, Mechanical and Structural Vibrations. Wiley, New York (1995)

6. T. Yang, Finite Element Structural Analysis. PrenticeHall, Englewood Cliffs, NJ (1986). 